\title{
Endoscopic ultrasound-guided transesophageal pericardiocentesis: an alternative approach to a pericardial effusion
}

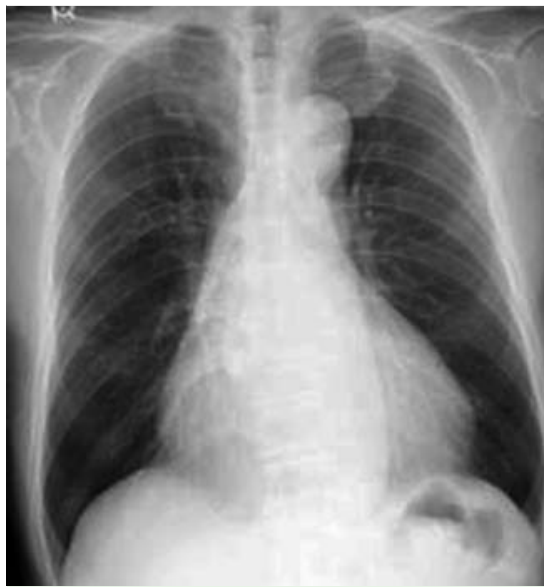

Fig. 1 Chest radiograph of a 76-year-old man who presented with chest discomfort showing a large cardiac shape.
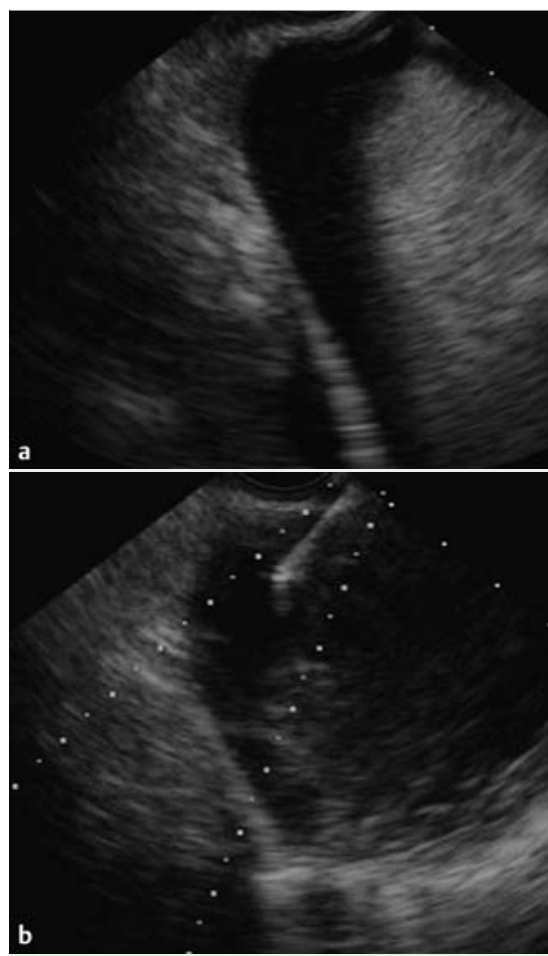

Fig. 3 Endoscopic ultrasound (EUS) image showing: $\mathbf{a}$ an anechoic fluid-filled space in the pericardial sac and the wide puncture window that could be identified away from cardiac movement; $\mathbf{b}$ the effusion being punctured from the esophagus and aspirated with a 22-gauge needle.

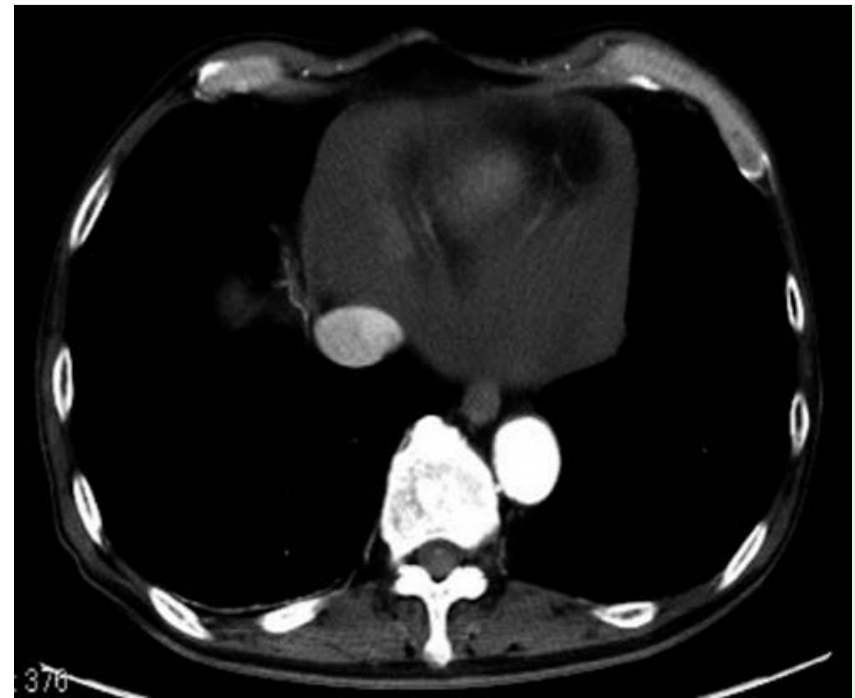

Fig. 2 Chest computed tomography (CT) scan showing a large volume pericardial effusion, most of which is located within the posterior sac.

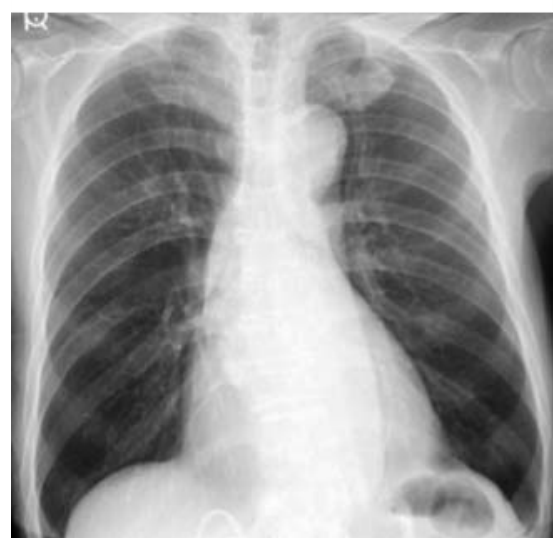

Fig. 4 Chest radiograph performed 2 months later showing a smaller cardiac shape.

Echocardiography-guided pericardiocentesis is a procedure commonly performed by cardiologist to evaluate pericardial effusions. It is generally considered a relatively safe procedure with minimal complications and a high success rate [1-3]. However, a safe approach to the pericardial effusion may on occasion not be possible. Endoscopic ultrasound (EUS) is capable of better visualization of the pericardium and left atrium. This is the first report to highlight the use of EUS-guided pericardiocentesis as an alternative method to the echocardiography-guided procedure.

A 76-year-old man presented with chest discomfort and suspected pericardial ef- fusion on a chest radiograph ( $\bullet$ Fig. 1 ). A computed tomography (CT) scan of his chest demonstrated a large volume pericardial effusion located in the posterior sac ( Fig.2). Conventional echocardiography-guided pericardiocentesis was attempted by an experienced cardiologist but was unsuccessful owing to the lack of effusion in the anterior sac, as had been previously shown on chest CT scan.

Having given his informed consent, the patient underwent an EUS-guided pericardiocentesis. With the patient in a supine position, a safe puncture route away from cardiac movement was identified ( $\bullet$ Fig.3a). The puncture was performed from the lower esophagus using a 22 -gauge needle ( Fig. $\mathbf{3}$ b) and $100 \mathrm{~mL}$ of fluid was successfully obtained. The fluid was clear and yellowish, with normal results on biochemical testing, negative cytology, and negative cultures for bacterial and acid-fast bacillus.

To reduce the risk of complications, intravenous antibiotics were given before the procedure to prevent procedural infection. During and after the procedure the patient was carefully monitored and remained free of chest discomfort. No early or late complications such as infection, arrhythmia, heart failure, or bleeding occurred. A repeat chest radiograph 2 months later revealed a smaller cardiac silhouette ( Fig.4). 
There are some cases that are challenging for percutaneous echocardiography-guided pericardiocentesis as it is not possible to obtain a clear puncture route because of the narrow window. EUS-guided pericardiocentesis may be a potential alternative if echocardiography-guided pericardiocentesis fails. Transbronchial pericardiocentesis has also been reported as an alternative method to echocardiographyguided pericardiocentesis [4].

Endoscopy_UCTN_Code_TTT_1AS_2AC

Competing interests: None

\section{Y. Hashimoto, K. Inoue}

Department of Gastroenterology, Fujigaoka Hospital Showa University, Yokohama, Japan

\section{References}

1 Tsang TS, Freeman WK, Sinak $L J$ et al. Echocardiographically guided pericardiocentesis: evolution and state-of-the-art technique. Mayo Clin Proc 1998; 73: 647-652

2 Tsang TS, Enriquez-Sarano M, Freeman WK et al. Consecutive 1127 therapeutic echocardiographically guided pericardiocenteses: clinical profile, practice patterns, and outcomes spanning 21 years. Mayo Clin Proc 2002; 77: 429-436

3 Tsang TS, Barnes ME, Hayes $S N$ et al. Clinical and echocardiographic characteristics of significant pericardial effusions following cardiothoracic surgery and outcomes of echo-guided pericardiocentesis for management: Mayo Clinic experience, 1979-1998. Chest 1999; 116: 322 - 331

4 Ceron L, Manzato M, Mazzaro F et al. A new diagnostic and therapeutic approach to pericardial effusion: transbronchial needle aspiration. Chest 2003; 123: 1753 -1758

\section{Bibliography}

Dol http://dx.doi.org/

10.1055/s-0032-1326074

Endoscopy 2016; 48: E71-E72

(c) Georg Thieme Verlag KG

Stuttgart · New York

ISSN 0013-726X

\section{Corresponding author}

\section{Y. Hashimoto, MD}

Gastroenterology, Internal Medicine Fujigaoka Hospital Showa University 1-39-35 Fujigaoka Aoba Yokohama 227-0043 Japan

Fax: +81-045-9726727

yusuke.h914@gmail.com 